\title{
Restrição alimentar no desempenho de machos do peixe beta (Betta splendens)
}

\author{
Elton Lima Santos*, Emerson Carlos Soares, Themis Jesus Silva, \\ Itairan Camelo de Macena Albuquerque, Sara Camylla de Souza Moura
}

Universidade Federal de Alagoas, Maceió, AL, Brasil

*Autor correspondente: e-mail, elton@zootecnista.com.br

\begin{abstract}
Resumo
Objetivou-se avaliar os efeitos da restrição alimentar sobre o desempenho de Bettasplendens. Foram utilizados 24 machos, com peso médio inicial de $0,55 \mathrm{~g} \pm 0,06$, distribuídos em 24 aquários experimentais (beteiras) de 1,5L de água,sem aeração disposto em um delineamento inteiramente casualizado, com quatro tratamentos e seis repetições.Os tratamentos foram: Tl - animais alimentados diariamente (controle), T2 - alimentados durante seis dias e um dia de restrição (6A/1R), T3 - alimentados durante cinco dias e dois dias de restrição (5A/2R) e T4 - alimentados durante quatro dias com três dias de restrição de ração (4A/3R). Os indivíduos foram alimentados duas vezes ao dia, às 08:00 e as 16:00h. As variáveis estudadas foram: Peso final (PF), Ganho de peso (GP), a conversão alimentar (CA), o consumo médio de ração (CMR), o Comprimento total (CTOTAL), Comprimento padrão (CPADRÃO), Índice de perfil (IPERFIL), fator de condição (FC) e altura.Os resultados foram submetidos a análise de variância (ANOVA), ocorrendo diferenças significativas entre as médias, estas foram comparadas pelo teste Tukey, a $5 \%$ de probabilidade. Não ocorreram diferenças significativas para os parâmetros: qualidade da água, sobrevivência, conversão alimentar e o índice de perfil. O melhor desempenho zootécnico foi encontrado no manejo alimentar de 6A/1R.
\end{abstract}

Palavras-chave: crescimento compensatório, estratégia alimentar, jejum, peixe ornamental,peixe de briga,

\section{Alimentary restriction on male betta fish (Betta splendens) performance}

\begin{abstract}
The aim of this study was to evaluate the effects of alimentary restriction on Betta splendens performance. For the experiment, 24 males were used, with initial average weight of $0.55 \pm 0.06$ grams, distributed in 24 experimental aquaria with 1.5 liters of water without aeration in a completely randomized design, with four treatments and six repetitions. The treatments were: $\mathrm{Tl}$ - daily fed animals (control), T2 - fed for six days and one day of restriction (6A/1R), T3 - fed for five days and two days of restriction (5A/2R) and T4 - fed for four days with three days of restriction (4A/3R). The fishes were fed twice a day at 08:00am and 4:00pm. The variables studied were: final weight (FW), weight gain (WG), alimentary conversion (FC), average feed consumption (CMR), total length (CTOTAL), standard length (CPADRÃO) profile index (IPERFIL), condition factor (CF) and height. The results were submitted to variance analysis (ANOVA), and the means were compared by Tukey's test at $5 \%$ of probability. No significant differences were observed for the following variables: water quality, survival, alimentary conversion and profile index. The best growth performance was observed when the feeding management of $6 \mathrm{~A} / 1 \mathrm{R}$ was applied.
\end{abstract}

Keywords: compensatory growth, fasting, feeding strategy, siamese fighting fish, ornamental fish 


\section{Introdução}

O mercado de peixes ornamentais é considerado um dos setores mais lucrativos da piscicultura brasileira e vem se expandindo rapidamente com o aumento na demanda mundial e as facilidades para exportação (Zuanon et al., 2011).

Um dos peixes de maior destaque da piscicultura ornamental brasileira é o peixe beta (Bettas plendens), também conhecido como peixe de briga ou internacionalmente como siamese fighting fish. Éconsiderada uma das espécies de ornamentais mais populares no Brasil e dentre as características favoráveis destes indivíduos destacam-se: a variedade de cores e nadadeiras, a respiração aérea acessória que permite sua manutenção em pequenos aquários sem aeração, a alta rusticidade e a elevada demanda no mercado (Lemos et al., 2014).

A otimização do manejo alimentar na piscicultura é um dos fatores preponderantes para quem busca o sucesso nesta atividade, ainda mais quando aproximadamente $70 \%$ dos custos de produção são advindos da alimentação (Santos et al., 2013). Deste modo, estratégias de manejo alimentar que empreguem a utilização de períodos curtos de restrição de alimento, visando a expressão do crescimento compensatório vêm sendo utilizado como forma de diminuir os custos de produção sem prejudicar o desempenho dos animais.

Um dos principais mecanismos de compensação de crescimento é a hiperfagia, que é uma alta taxa de consumo alimentar, fenômeno que pode ser observado em peixes sendo realimentados após um período de privação alimentar (Ribeiro\&Tsuzuki, 2010).Assim, tanto estratégias de alimentação com apenas uma fase de restrição alimentar e realimentação, como vários ciclos de restrição e realimentação, têm sido usadas para promover respostas compensatórias no desempenho produtivo de peixes (Palma et al., 2010).

Segundo Eroldogan et al. (2008), ciclos de arraçoamentos, incluindo poucos dias de jejum, podem compensar pela redução dos custos com ração e mão-de-obra, os estudos deAbdelHakim et al. (2009) com juvenis híbridos de tilápia (Oreochromis niloticus x Oreochromis aureus),
Wang et al. (1998) com Lepomis cyanellus, Souza et al. (2003) com juvenis de pacu (Piaractus mesopotamicus), Graeff et al. (2003) com carpa comum (Cyprinus carpio), Oh et al. (2007) com Pagrus major e por Blanquet\& Oliva-Teles (2010) com Scophthalmus maximus L., reforçam este argumento.

Estudos relacionados com ganho compensatório após períodos de privação alimentar de peixe de produção para consumo são bastante difundidos, entretanto, pesquisas envolvendo o manejo alimentar de peixes ornamentais e particularmente o Betta, são incipientesdevido comportamento territorialista e agressivo (Dzieweczynski \& Leopard, 2010), envolvendo maiores cuidados no manejo, o que aumenta muito o dispêndio de trabalho para o arraçoamento, pelas exigências de ambientes individuais.

O objetivo desta pesquisa foi avaliar períodos de restrição alimentar e realimentação sobre o desempenho de Bettasplendens.

\section{Material e Métodos}

Durante um período de avaliação de desempenho de 42 dias, foram utilizados 24 alevinos de Bettasplendens, machos, com idade aproximada de 35 dias, com peso médio inicial de 0,55g $\pm 0,06$ e comprimento total de $5,13 \mathrm{~cm}$, sendo todos os animais provenientes da mesma desova, advindos de uma piscicultura comercial de Maceió, Alagoas, Brasil.

Os animais foram alojados individualmente em 24 beteiras (aquários), com capacidade efetiva de 1,5L de água. Inicialmente foi realizado um período de seis dias de aclimatação, antes do período de avaliação experimental.

As estratégias de alimentação utilizadas foram: peixes alimentados diariamente (controle), peixes alimentados em ciclos compostos por seis dias de alimentação seguidos de um dia de restrição dealimento (6A/1R), peixes alimentados por cinco dias de alimentação seguidos de dois dias de restrição dealimento $(5 \mathrm{~A} / 2 \mathrm{R})$ e peixes alimentados em cicloscompostos por quatro dias de alimentação seguidosde três dias de restrição de alimento (4A/3R), correspondendo aos quatro tratamentos experimentais, com seis 
repetições cada tratamento. Sendo que cada unidade experimental (beteira) foi uma parcela experimental com um peixe cada.

Em todos os tratamentos foi utilizada ração comercial com $56 \%$ de PB em todos os tratamentos (Tabela 1), até a aparente saciedade. A ração foi ofertada duas vezes ao dia pela manhã e tarde -08h30min e 15h30min.

Tabela 1. Composição percentual da ração comercial $56 \%$ de PB.

\begin{tabular}{ccc}
\hline Níveis de garantia & $(\%)$ & Ração comercial* \\
\hline Umidade & (MAX.) & $12,00 \%$ \\
\hline Proteína bruta & (MIN.) & $56,00 \%$ \\
Extrato etéreo & (MIN.) & $5,50 \%$ \\
Matéria mineral & (MAX.) & $12,00 \%$ \\
Matéria fibrosa & (MAX.) & $6,00 \%$ \\
Cálcio & (MAX.) & $2,50 \%$ \\
Fósforo & (MIN.) & $0,70 \%$ \\
\hline
\end{tabular}

Níveis de garantia por $\mathrm{kg}$ do produto: vit. $\mathrm{A}=27.000,00 \mathrm{Ul}$; vit.D3 = 5.400,00 Ul; vit. E $180,00 \mathrm{mg}$; vit. $K 3=9,00 \mathrm{mg}$; vit. $B 1=9,00 \mathrm{mg}$; vit. $B 2=18,00 \mathrm{mg}$; vit. $B 6=18,00 \mathrm{mg}$; vit. $B 12=18,00 \mathrm{mcg}$; vit. $C=350,00 \mathrm{mg}$; ácido pantotênico $=72,00 \mathrm{mg}$; biotina $=0,90 \mathrm{mg}$ niacina= 270,00 mg; $\mathrm{Fe}=18,00 \mathrm{mg} ; \mathrm{Cu}=72,00 \mathrm{mg} ; \mathrm{Mn}=225,0 \mathrm{mg} ; \mathrm{Zn}=75,00 \mathrm{mg} ; \mathrm{I}=$ $36,00 \mathrm{mg}$ e $\mathrm{Se}=0,90 \mathrm{mg}$

Diariamente foi realizada a troca parcial da água ( $\pm 20 \%$ do volume total), com a retirada das excretas e sobras de ração do fundo dos aquários através de mangueira de pipetagem.

Os indicadores de qualidade de água: $\mathrm{pH}$, oxigênio e temperatura foram monitorados diariamente,em dois horários (8:00 e 16:00 horas), através de sonda multiparâmétrica da marca HANNA Instruments, modelo 9828, Woonsocket, USA, a uma profundidade de aproximadamente $10 \mathrm{~cm}$ abaixo da lâmina d'água. A amônia total $\left(\mathrm{NH}_{3}+\mathrm{NH}_{4}\right)$ foi mensurada, em avaliações a cada dois dias, com o auxílio de espectrofotômetro da marca HANNA Instruments, modelo HI 83203, Bélgica, utilizando os reagentes de modelo HI93700-01. O nitrito (NO2-) e o fosfato (P) dos efluentes foram mensurados com este mesmo equipamento utilizando os reagentes HI93707-01 para o nitrito e H193717-01 para o fosfato.

No início e no final do experimento todos os animais foram submetidos a biometria, onde as variáveis avaliadas foram: peso final (PF), ganho de peso (GP= peso final - peso inicial), comprimento total (COMTOTAL) medido entre a parte inicial da cabeça até o final da nadadeira caudal, o comprimento padrão (COMPADRÃO) compreendendo a medida anterior da cabeça até $O$ início da nadadeira caudal, a altura (ALTURA), o índice de perfil (IPERF= comprimento padrão/altura), $\mathrm{o}$ fator de condição ( $F C=$ peso final/comprimento total ${ }^{3} \times 100$ ), consumo médio de raçãoeconversão alimentar ( $C A=$ consumo de ração /ganho de peso total).

O experimento foi conduzido num Delineamento inteiramente casualizado, com quatro tratamentos e seis repetições, onde, todos os dados obtidos de desempenho foram submetidos a análise de variância (ANOVA, 5\% de confiabilidade) e quando os parâmetros foram significativos, estes foram submetidos ao teste de comparação de médias de Tukey ao nível de $5 \%$ de probabilidade, utilizando-se - programa estatístico computacional SAEG 9.0(UFV, 2005).

\section{Resultados e Discussão}

Durante o período experimental, os parâmetros de qualidade de água mantiveramse dentro da faixa ideal para aquicultura, conforme preconizado por Boyd (2000) com valores médios de temperatura, $\mathrm{OD}, \mathrm{pH}$, amônia total enitrito de: $26,2 \pm 0,8{ }^{\circ} \mathrm{C} ; 6,4 \pm 1,0 \mathrm{mg} / \mathrm{L}$; $7,0 \pm 0,2 ; 0,20 \pm 0,05 \mathrm{mg} / \mathrm{L} ; 0,009 \pm 0,001 \mathrm{mg} / \mathrm{L}$ respectivamente.

Ao final de 40 dias de avaliação, os peixes submetidos aos tratamentos $6 \mathrm{~A} / 1 \mathrm{R}$ e $5 A / 2 R$ apresentaram peso finais semelhantes aos peixes continuamente alimentados (controle). No entanto, esse resultado não foi observado para as outras variáveis, pois, a estratégia alimentar $4 \mathrm{~A} / 3 \mathrm{R}$ resultou em peixes com peso final inferiores aos peixes continuamente alimentados, mas que não diferiu significativamente do tratamento com 5A/2R (Tabela 2).

Corroborando com os resultados encontrados no presente estudo, Palmaet al. (2010) trabalhando com juvenis de tilápia do Nilo da linhagem GIFT, verificaram queexemplares submetidos à estratégia $5 \mathrm{~A} / 2 \mathrm{R}$ apresentaram peso e comprimento final semelhantes aos peixes sem restrição alimentar, fato que não foi observado para os peixes submetidos a estratégia de manejo alimentar 4A/3R, que resultou em peixes com peso final e comprimento final inferiores, ou seja, foram diferentes significativamente $(p<0,05)$.

Animais submetidos a maior período de privação alimentar (4A/3R) apresentaram 
resultados inferiores de comprimento padrão, contudo não foi observada diferença significativa entre os demais tratamentos.

O fator de condição revelou que os bettas machos que passaram por períodos de privação alimentar obtiveram melhores médias e com diferenças significativas $(p<0,05)$ em relação a exemplares que não foram submetidos à restrição alimentar.

Tabela 2. Desempenho produtivo de machos de Betta splendens submetidos à restrição alimentar e realimentação.

\begin{tabular}{|c|c|c|c|c|c|}
\hline Variáveis & $7 A / O R$ & $6 \mathrm{~A} / 1 \mathrm{R}$ & $5 \mathrm{~A} / 2 \mathrm{R}$ & $4 \mathrm{~A} / 3 \mathrm{R}$ & $\mathrm{CV}(\%)^{*}$ \\
\hline $\mathrm{PI}(\mathrm{g})$ & $0,55 \pm 0,08$ & $0,55 \pm 0,07$ & $0,55 \pm 0,08$ & $0,55 \pm 0,07$ & 13,89 \\
\hline PF (g) & $1,94 \pm 0,41 \mathrm{~A}$ & $1,84 \pm 0,24 \mathrm{~A}$ & $1,54 \pm 0,14 A B$ & $1,30 \pm 0,13 \mathrm{~B}$ & 15,55 \\
\hline GP (g) & $1,39 \pm 0,42 \mathrm{~A}$ & $1,29 \pm 0,23 A B$ & $0,99 \pm 0,08 \mathrm{BC}$ & $0,75 \pm 0,10 \mathrm{C}$ & 22,32 \\
\hline $\mathrm{CT}(\mathrm{cm})$ & $5,71 \pm 0,44 \mathrm{~A}$ & $4,75 \pm 0,22 A B$ & $5,18 \pm 0,37 \mathrm{~B}$ & $4,88 \pm 0,39$ B & 7,12 \\
\hline $\mathrm{CP}(\mathrm{cm})$ & $3,81 \pm 0,28 \mathrm{~A}$ & $3,64 \pm 0,14 \mathrm{~A}$ & $3,54 \pm 0,17 \mathrm{~A}$ & $3,21 \pm 0,20 \mathrm{~B}$ & 5,71 \\
\hline Altura $(\mathrm{cm})$ & $1,41 \pm 0,14 \mathrm{~A}$ & $1,27 \pm 0,06 \mathrm{AB}$ & $1,15 \pm 0,09 \mathrm{~B}$ & $1,15 \pm 0,05 B$ & 7,29 \\
\hline IPERF & $2,93 \pm 0,59$ & $2,87 \pm 0,09$ & $3,09 \pm 0,15$ & $2,80 \pm 0,18$ & 12,75 \\
\hline FC & $1,04 \pm 0,16 \mathrm{~A}$ & $1,71 \pm 0,10 \mathrm{~B}$ & $1,14 \pm 0,27 \mathrm{~B}$ & $1,15 \pm 0,24 \mathrm{~B}$ & 16,12 \\
\hline CMR (g) & $1,93 \pm 0,25 \mathrm{~A}$ & $1,79 \pm 0,14 A B$ & $1,55 \pm 0,15 \mathrm{~B}$ & $1,51 \pm 0,30 \mathrm{~B}$ & 13,04 \\
\hline CA & $1,51 \pm 0,47$ & $1,43 \pm 0,29$ & $1,57 \pm 0,11$ & $2,07 \pm 0,48$ & 24,53 \\
\hline
\end{tabular}
condição; $C M R=$ consumo médio de ração; $C A=$ conversão alimentar.

Através do fator de condição podeseacompanhar o grau de atividade alimentar de umaespécie, verificando se ela está ou não fazendo bom usoda fonte nutricional (Souza et al., 2003). Assim, o presente estudo confirma que para os animais que foram submetidos a algum tipo de manejo com restrição alimentar não foram observadas diferenças significativas $(p>0,05)$.

O crescimento compensatório pode ser obtido por hiperfagia (Wang et al., 2000) ou dos efeitos somados de hiperfagia e aumento na eficiência de crescimento (Qian et al., 2000). Desta forma, oconsumo médio de ração (CMR), o comprimento total (CT) e a altura dos animais submetidos ao tratamento controle obtiveram resultados superiores quando comparado aos valores dos tratamentos $5 \mathrm{~A} / 2 \mathrm{R}$ e $4 \mathrm{~A} / 3 \mathrm{R}$, porém não havendo diferença significativa $(p>0,05)$ para o tratamento em que os animais passaram por um dia de privação de alimento. Conforme observadopor Morshediet al. (2013), trabalhando com o peixe ornamental Poecilia latipinna, indivíduos submetidos a períodos curtos de restrição alimentar e posterior realimentação tendem a consumir mais ração após a realimentação, que não expressam efeito significativo sobre o consumo do período total experimental e sobre o desempenho zootécnico.

Para os resultados de comprimento padrão foi verificado que os animais submetidos a maior período de restrição alimentar apresentaram resultados inferiores, contudo não foi observada diferença significativa $(p>0,05)$ entre os demais tratamentos do presente estudo.

Em relação aos valores encontrados de CA e IPERF, estes não apresentaram diferenças significativas entreos diferentes tratamentos. Semelhantes resultados foram observados por Graeff et al. (2003) para CA de carpas comum (Cyprinus carpio L.), ondenãoocorrevefeito significativo dos tratamentos, submetidos a restrição alimentar.

Arauco \& Costa (2012) trabalhando com juvenis de tilápia do Nilo, relataram, que a restrição alimentar diminui o desempenho produtivo dos peixes, segundo os mesmos autores, nem a qualidade da água e nem a conversão alimentar foram influenciados pela privação de hiperfagia e levando a ocorrência de ganho compensatório após a realimentação, consequentemente um melhor aproveitamento dos nutrientes.

Zaúldua \& Naya (2014) relatam que a melhor eficiência de utilização dos nutrientes dos animais quando submetidos a períodos de inanição, podem estar relacionados às estratégias fisiológicas dos peixes, que possuem comparativamente aos mamíferos e aves, taxas de renovação celular das células intestinais (enterocitos) relativamente menores, o que provavelmente está relacionado a regulação 
do trato digestivo e diminuição física (Gaucher et al., 2012), durante períodos de restrição alimentar.

Deste modo, Eslamloo et al. (2012) estudando $\circ$ efeito da privação alimentar $e$ realimentação em Barbonymus schwanenfeldii afirma que estratégias curtas de até quatro dias de restrição de ração e posterior realimentação por doze dias pode ser vantajoso do ponto de vista produtivo e econômico, pois, encontrou resultados melhores com relação ao desempenho zootécnico desses animais.

Estratégias de manejo de alimentação que realizem curtos períodos de privação de ração seguidos de posterior realimentação até a aparente saciedade, pode ser uma estratégia promissora para diminuir os custos de produção do cultivo de Betta splendens, minimizando os custos com mão de obra para o arraçoamento e ofertando menores quantidades de ração melhorando o manejo em sistemas de cultivo com densidades de um indivíduo por unidade experimental.

\section{Conclusões}

Nas condições estudadas a estratégia de manejo alimentar de 6A/1R pode ser aplicada como indicativo no cultivo de Betta splendens sem prejuízos ao desempenho do animal.

\section{Referências}

Abdel-Hakim, N.F., State, H.A.A., Al-AzabA.A., El-Kholy, F. 2009. Effect of Feeding Regimes on Growth Performance of Juvenile Hybrid Tilapia (Oreochromis niloticusx Oreochromis aureus). World Journal of Agricultural Sciences 5: 49-54.

Arauco, L.R.R., Costa, V.B. 2012. Restrição alimentar no desempenho produtivoda tilápia (Oreochromis niloticus). Comunicata Scientiae 3: 134-138.

Blanquet, I.,Oliva-Teles, A.2010. Effect of feed restriction on the growth performance of turbot (Scophthalmus maximus L.) juveniles under commercial rearing conditions. Aquaculture Research 41: 1255-1260.

Boyd, C.E. 2000. Water Quality, An Introduction. Kluwer Academic Publishers, Boston, USA.330 p.

Dzieweczynski, T.L, Leopard, A.K. 2010. The effects of stimulus type on consistency of responses to conflicting stimuli in Siamese fighting fish. Behavioral Processes 85: 83-89.
Eroldogan, O.T., Tasbozan,O., Tabakoglu, S. 2008. Effects of restricted feeding regimes on growth and feed utilization of juvenile Gilthead Sea bream, Sparus aurata. Journal of the World Aquaculture Society 39: 267-274.

Eslamloo,K., Morshedi, V., Azodi, M.,Ashouri, G., Ali,M., labal,F. 2012.Effects of Starvation and Re-Feeding on Growth Performance, Feed Utilization and Body Composition of Tinfoil Barb (Barbonymus schwanenfeldii). World Journal of Fish and Marine Sciences 4: 489-495,

Gaucher, L., Vidal, N., D'Anatro, A., Naya, D. E. 2012. Digestive flexibility during fasting in the characid fish Hyphessobrycon luetkenii. Journal of Morphology 273: 49-56.

Graeff, A., Pruner, E.N., Mondardo, M. 2003. Desempenho das carpas comum (Cyprinus carpio L.) em um programa de restrição alimentar na fase de alevinos. Anais do Congresso Iberoamericano Virtual 1: 420-426.

Lemos, M.V.A., Arantes, T.Q., Souto, C.N., Martins, G.P., Araujo, J.G., Guimarães, I.G. 2014. Efeito da relação energia: proteína digestível no crescimento e composição química de carcaça em dietas para alevinos de Betta splendens. Ciência e Agrotecnologia 38: 76-84.

Morshed, V., Kochanian, P., Ahmadi-Niko, M., Azodi, M., Pasha-Zanoosi, H.2013. Compensatory growth response of sailfin molly, Poecilia latipinna (Lesueur, 1821) to starvation and refeeding. International Journal of Aquatic Biology 1 (3): 109115.

Oh, S.Y., Choong, H.N., Cho, S.H. 2007. Effect of Restricted Feeding Regimes on Compensatory Growth and Body Composition of Red Sea Bream, Pagrus major. Journal of the World Aquaculture Society 38: 443-449.

Palma, E.H., Takahashi, L.S., Dias, L.T.S., Gimbo, R.Y., Kuyima, T.J., Nicodemo, D. 2010. Estratégias de alimentar com ciclos de restrição e realimentação no desempenho produtivo de juvenis de tilápia do Nilo da linhagem GIFT. Ciência Rural 4: 421- 426.

Qian, X., Cui, Y.,Xiong, B.; Yang, Y. 2000. Compensatory growth, feed utilization and activity in gibel carp, following feed deprivation. Journal of Fish Biology 56: 228-232.

Ribeiro, F.F., Tsuzuki, M.Y. 2010. Compensatory growth responses in juvenile fat snook, Centropomus parallelus Poey, following food deprivation. Aquaculture Research 41: 226-233.

Santos, E.L.,Silva, F.C.B., Pontes, E.C., Lira,R.C., Cavalcanti, M.C.A. 2013. Resíduo do processamento do extrato de própolis vermelha em ração comercial para alevinos de Tilápia 
do Nilo (Oreochromis niloticus). Comunicata Scientiae 4(2): 179-185.

Souza, V.L., Urbinati, E.C., Martins, M.I.E.G., Silva, P.C. 2003. Avaliação do Crescimento e do Custo da Alimentação do Pacu (Piaractus mesopotamicus Holmberg, 1887) Submetido a Ciclos Alternados de Restrição Alimentar e Realimentação. Revista Brasileira de Zootecnia 32: 19-28.

Universidade Federal De Viçosa - UFV. Sistema de análise estatística e genética (SAEG 9.0). Central de processamento de dados. Viçosa, 2005.

Wang, N., Hayward, R.S., Noltie, D.B. 1998. Effect of feeding frequency on food consumption and growth, size variation, and feeding pattern of age- 0 hybrid sunfish. Aquaculture 165: 261-267.

Wang, Y., Cui, Y., Yang, Y.,Cai, F. 2000. Compensatory growth in Hybrid tilapia, Oreochromis mossambicus $\times$ O. niloticus, reared in sea water. Aquaculture 189: 101-108

Zaldúa, N.,Naya, D.E. 2014. Digestive flexibility during fasting in fish: A review. Comparative Biochemistry and Physiology Part A: Molecular \& Integrative Physiology 169: 7-14.

Zuanon, J.A.S., Salaro, A.L., Furuya, W.M. 2011. Produção e nutrição de peixes ornamentais. Revista Brasileira de Zootecnia 40: 165-174. 
Produção e Saúde Animal

Com. Sci., Bom Jesus, v.7, n. 1, p.12-23, Jan./Mar. 2016 
Santos et al. (2016) / Restrição alimentar no desempenho de machos...

Com. Sci., Bom Jesus, v.7, n.1, p.12-23, Jan./Mar. 2016 
Produção e Saúde Animal

Com. Sci., Bom Jesus, v.7, n. 1, p.12-23, Jan./Mar. 2016 
Santos et al. (2016) / Restrição alimentar no desempenho de machos...

Com. Sci., Bom Jesus, v.7, n.1, p.12-23, Jan./Mar. 2016 
Produção e Saúde Animal

Com. Sci., Bom Jesus, v.7, n. 1, p.12-23, Jan./Mar. 2016 
Santos et al. (2016) / Restrição alimentar no desempenho de machos...

Com. Sci., Bom Jesus, v.7, n.1, p.12-23, Jan./Mar. 2016 